\title{
Retroperitoneoscopic Nephrectomy in Benign Pathology
}

\author{
Rodrigo S. Quintela, Leonardo R. Cotta, Marcelo F. Neves, David L. Abelha Jr, Jose E. \\ Tavora
}

Section of Urology, Hospital da Previdencia dos Servidores do Estado, Belo Horizonte, Minas

Gerais, Brazil

\begin{abstract}
Introduction: We report our experience with 43 retroperitoneal laparoscopic nephrectomy for benign kidney disease. Materials and Methods: All patients had a poor function from obstructive uropathology and renal atrophy. None of these patients had a previous lumbotomy. Retroperitoneoscopy was performed with 4 trocar port technique in a lateral position. The retroperitoneal space is created by using a Gaur's balloon made of sterile glove. The approach to vascular pedicle was done posteriorly and vessels were clipped by metal and Hem-o-lock (Weck Closure Systems, North Carolina, USA) clips. The sample was intact extracted in an Endo-Bag prolonging one trocar incision.

Results: Median operative time was 160 minutes and median blood loss was $200 \mathrm{~mL}$. Four cases (9\%) were converted to open surgery: one case due to bleeding and 3 cases due to technical difficulties regarding perirenal adherences. Most patients (39) checked out from the Hospital in day two. Four of them were left over 3 days due to wound complications. Conclusions: Retroperitoneoscopy offers a safe, effective and reproductive access to nephrectomy for benign pathologies.
\end{abstract}

Key words: kidney; nephrectomy; laparoscopy; retroperitoneal space

Int Braz J Urol. 2006; 32: 521-8

\section{INTRODUCTION}

Laparoscopic nephrectomy was first successfully performed by Clayman in 1990 using the transperitoneal route (1). The pure retroperitoneal access for nephrectomy was described by Gaur three years later, using a balloon to create the surgical working space $(2,3)$. Despite the preference for retroperitoneal approach in open urologic surgery worldwide the transperitoneal approach is the preferred technique for laparoscopic urologic surgery. Furthermore direct retroperitoneal access (retroperitoneoscopy) is attracting more interest and application in urology, becoming the preferential approach for nephrectomy in many expertise laparoscopic centers (4-9). This data de- scribes our experience with retroperitoneoscopic simple nephrectomy.

\section{MATERIALS AND METHODS}

Forty-three retroperitoneal laparoscopy nephrectomies were performed from November 2003 through 2005 for benign kidney disease. Our initial experience started with retroperitoneal approach. This data show patients aged between 25-75 years, mean age was 47 years being 15 male and 28 females. Twenty nephrectomies (46\%) were performed in right kidneys and 23 cases in the left kidney (54\%). Symptomatology was presented as lumbar pain $(56 \%)$, 
pyelonephritis in 16 cases (37\%) and three cases (7\%) with renovascular hypertension. The work up was done in all cases following ultrasound, intravenous pyelogram (IVP) or axial computerized tomography and scint scan. Thirty-three patients (76\%) presented hydronephrosis with no renal function and the other 10 patients (24\%) presented renal atrophy. The etiology of the renal damage is showed in the Table-1. Three of the patients presented horseshoe kidneys with UPJ (ureteral pelvic junction) syndrome. Two patients had previous lumbar urologic interventions (percutaneous nephrolithotripsy and a percutaneous nephrostomy).

\section{Surgical Technique}

The patient is placed in lateral position as in an open surgery (classical lumbotomy position) with general anesthesia. In an attempt to get more room to face up the kidney, we flexed the table. The camera assistant stays at the surgeon's side at a cephalic position and the second assistant stays at the opposite side. A transverse incision $(15 \mathrm{~mm})$ is made just below the 12th costal arc and the muscles are dissected until the toracolumbar fascia is opened achieving the retroperitoneal space. A digital dissection is made in the retroperitoneal space pulling the peritoneum anteriorly and displacing the fat from this body wall. A Gaur balloon (Figure-1) is placed in the space and filled with $800 \mathrm{~mL}$ of saline solution. The Hasson trocar is placed and fixed at the body wall to avoid air linkage. $\mathrm{ACO}_{2}$ insufflation is performed at a pressure of $12 \mathrm{~mm}$. A $0^{\circ}$ laparoscopic lent is introduced

Table 1 - Etiology of the lost renal function.

\begin{tabular}{lc}
\hline Pathology & Patients (\%) \\
\hline Ureteropelvic junction stricture & $18(42 \%)$ \\
Ureteral calculi & $12(27.7 \%)$ \\
Ureteral trauma & $3(7 \%)$ \\
Renal tuberculosis & $3(7 \%)$ \\
Congenital renal atrophy & $3(7 \%)$ \\
Renovascular hypertension & $3(7 \%)$ \\
Renal trauma & $1(2.3 \%)$ \\
Total & $43(100 \%)$ \\
\hline
\end{tabular}

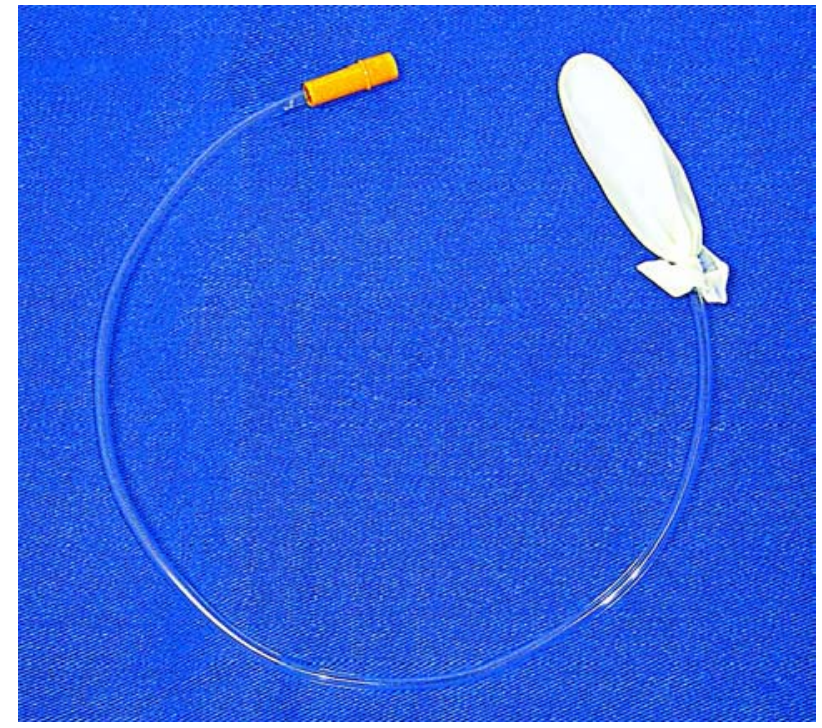

Figure 1 - Gaur's balloon made of a Nelaton $16 \mathrm{~F}$ catheter and a finger glove.

by the Hasson port and the other trocars are placed under direct vision: a $10 \mathrm{~mm}$ trocar is placed $2 \mathrm{~cm}$ above the iliac crest and two $5 \mathrm{~mm}$ trocars are placed in anterior axillary line (one next to the costal arc and the other by the iliac crest) (Figure-2).

The psoas muscle is the main anatomic landmark. This muscle is dissected and the ureter and the

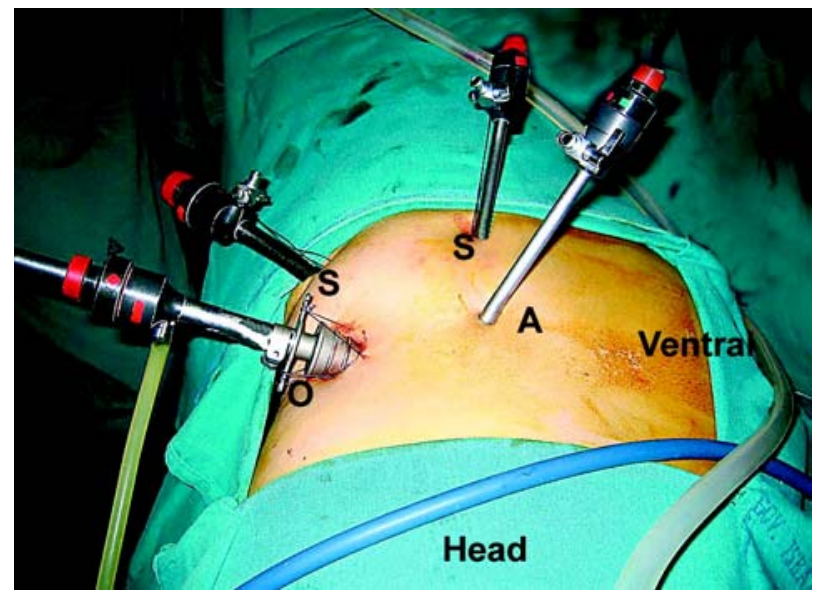

Figure 2 -Superior view of trocar port incisions for a left side retroperitoneoscopic nephrectomy. The Hasson trocar is fixed with sutures. The lens port $(O)$, the surgeon ports $(S)$ and the assistant port (A) are signaled. 
gonad vein in the left or the inferior vena cava in the right are identified. The renal vein is dissected in the right side following the vena cava and the renal artery is dissected over the renal vein. In the left side, the renal vein is identified following the gonad vein and the artery is found just above the renal vein. Metal clips are used to occlude the artery and a Hem-o-lock ( is used at the renal vein. After the vascular control, the dissection is initiated inside the Gerota's fascia by the renal upper pole preserving the adrenal gland in most of the cases. The lower pole then is dissected and the ureter is clip occluded with a metal clip. In cases of horseshoe kidney, the renal isthmus is coagulated with an electrocautery and laparoscopically sutured with absorbed suture if necessary. In voluminous hydronephrotic kidneys the fluid in the collecting system is aspirated to facilitate perirenal dissection. The specimen is entrapped in a handmade bag. In cases where the samples are small, we utilize the first $15 \mathrm{~mm}$ lumbar incision to remove the specimen and with larger samples we prolong the inguinal port incision making a small Gibson's incision (Figure3 ). A Penrose drain is placed in cases of infected kidney.

\section{RESULTS}

All of the surgeries were performed by the same surgeon or under his supervision. Table-2 resumes the results. Median operative time was 160 minutes (120 to 240 minutes) with average estimated blood loss of $200 \mathrm{~mL}$. Blood transfusion was not necessary in any of the cases. Three patients presented intra surgical bleeding during pedicle dissection. Two patients with renal vein bleeding were laparoscopic controlled with a Hem-o-lock clip close to the vena cava. Another bleeding from an injury at the right adrenal vein required conversion to open surgery.

There were no major complications. Six patients developed abdominal body wall complications: three patients developed subcutaneous emphysema with no clinical repercussion and a spontaneous resolution; two patients developed surgical wound hematoma in the first trocar incision and one patient

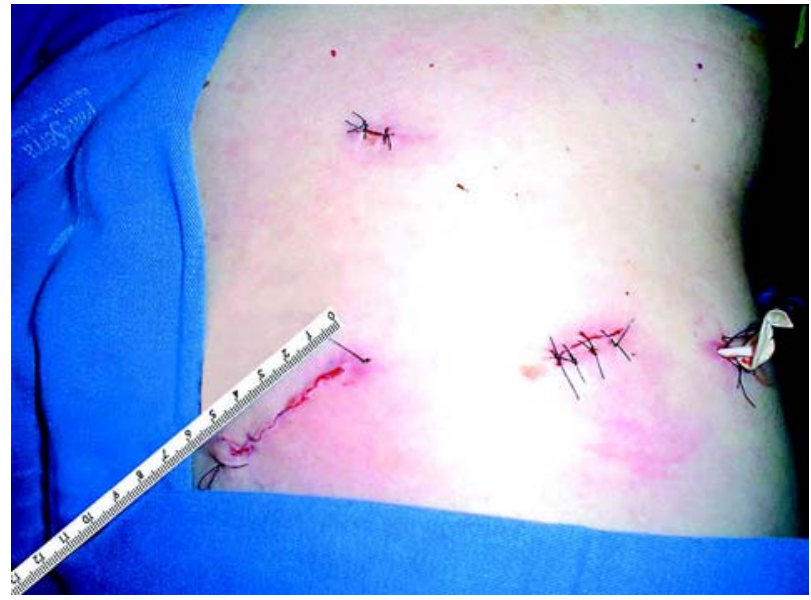

Figure 3 - Final aspect of the incisions. The specimen was removed by the $5 \mathrm{~cm}$ inguinal incision. A Penrose drain is left in the dorsal surgeon working port.

developed surgical wound infection. All of those patients were treated clinically.

Conversion was necessary in 4 cases (9\%). One patient due to hemorrhage and three patients due to technical difficulties. All of them presented chronic or tuberculous pyelonephritis and perirenal adherence. In none of the cases surgical reintervention was needed.

Diet was initiated 6 hours after surgery. Most of the patients (39) were released from the hospital the second day after surgery. Four patients were left in the hospital for more than 48 hours due to surgical wound complications.

Histopathological diagnosis confirmed three cases of renal tuberculosis and 40 cases of renal atrophy with or without chronic inflammatory process.

Table 2 - Surgical results.

\begin{tabular}{lc}
\hline Patients & $\mathbf{4 3}$ \\
\hline Operative time (median) & 160 minutes \\
Blood lost & $200 \mathrm{ml}$ \\
Conversion (\%) & $4(9 \%)$ \\
Major complication (\%) & 0 \\
Minor complication (\%) & $6(13 \%)$ \\
Hospital stay & 2 days \\
\hline
\end{tabular}




\section{COMMENTS}

Laparoscopic nephrectomy is widely performed by the transperitoneal approach. After retroperitoneal technique description by Gaur these has become the preferential approach in outstanding centers for the surgical treatment of most kidney pathologies even renal tumors (4-9).

The advantage of retroperitoneoscopy are the preservation of the peritoneal cavity and the posterior access to the renal pedicle making possible a straight dissection and the control of the vessels in the first step of the surgery. This approach can be done without any difficulties even in hard cases $(10,11)$.

The main disadvantage of retroperitoneoscopy is the reduced working space requiring a better synchronized surgical team to avoid instrument collision. The use of Gaur's balloon creates a large space, which allow the surgeon to get enough room reaching all the kidney limits.

The main contraindication of retroperitoneoscopy is the presence of previous lumbotomy. Relative contraindication because of technical aspects should be attempt in patients with chronic inflammatory pathologies such as renal tuberculosis or xantogranulomatous pyelonephrosis (10-12). In those cases, the possibility of conversion is higher because of the adherences. In 3 cases of this series, patients with a chronic inflammatory process (one with renal tuberculosis) were submitted to conversion to open surgery due to technical difficulties. Furthermore, in two patients with tuberculosis the surgery was performed without any difficulties or complications. Some reported series show a significant number of cases of renal tuberculosis successfully treated by retroperitoneoscopy $(10,11)$. Although it presents a better chance of conversion, the presence of a chronic inflammatory process is not an absolute contraindication for laparoscopic nephrectomy. In such cases, transperitoneal or hand-assisted laparoscopy should be a better option.

Previous minimally invasive lumbar procedures such as nephrostomy and percutaneous nephrolithotripsy do not exclude retroperitoneoscopy $(6,7,13)$. In two patients of this series with previous history of nephrostomy and percutaneous nephrolithotripsy, the procedure was performed without any difficulties.

In patients with horseshoe kidney the presence of anomalous vessels require an extra careful vascular approach (3). In three patients of this series with horseshoe kidney retroperitoneal laparoscopic nephrectomy was performed without difficulties in reaching the renal pedicle but a longer operative time was needed.

The incidence of vascular lesions in transperitoneal urological laparoscopic surgery vary between $1.5 \%$ to $2 \%$ in large series $(14,15)$. Although there is not much data for retroperitoneal approach its reported incidence is similar (16). In this data we had three cases of vascular bleeding during pedicle dissection. In one of the cases a left adrenal vein bleeding required conversion to open surgery. Conversion was not required in two other cases of renal pedicle bleeding. Conversion to open surgery depends on the extension of the vascular lesion and of the surgeon skills to control bleeding. A temporary increase of inflation pressure up to 15 or $20 \mathrm{mmHg}$ reduces venous hemorrhage in the retroperitoneal space and a vascular control maneuver must be considered. In one case it was necessary the placement of another trocar port for a better exposition. We experienced no bleeding problems using metal clips for renal artery control and Hem-o-lock (Weck Closure Systems, North Caroline, USA) clips for renal vein control.

Emphysema is the most common abdominal wall complication added to hematoma and wound infection (17). Hematoma at the place of the trocar port is more often seen in retroperitoneal laparoscopy rather than in transperitoneal laparoscopy. The reason for that is the thick layer of lumbar muscle that offers a higher tension in mobilizing instruments. This problem can be reduced by choosing the right place for the trocar introduction in the body wall avoiding paravertebral muscle and a close proximity to the bone structure such as costal arc and iliac crest. In this data we had two cases of wound hematoma with a spontaneous resolution and one case of wound infection treated clinically. The use of a blunt tip cannula (Auto Suture-Menlo Park, California) not requiring fixation to the body wall with sutures can avoid this complication. 
The $\mathrm{CO}_{2}$ inflation can cause subcutaneous emphysema, especially pneumothorax and pneumomediastine if the retroperitoneal pressure reach levels over $15 \mathrm{mmHg}$ (18). Usually no important clinical repercussion is seen on those patients (19). A pressure of $12 \mathrm{mmHg}$ gives the surgeon a good working space. Three cases of subcutaneous emphysema with no clinical repercussion were observed in this data.

Total surgical conversion frequency was $9 \%$. Most of those conversions occurred due to hemorrhage or technique difficulties caused by perirenal adherence. The data literature shows up to $10 \%$ of conversion for simple laparoscopic nephrectomy $(6,9)$. The majority of conversions occur due to inflammatory processes or previous surgeries (11). When surgical conversion becomes necessary it can be rapidly performed by lumbotomy, prolonging the first trocar incision that allows the surgeon to continue with the conventional lumbar incision with good cosmetic results.

The sample extraction can be performed by a new incision, amplifying the trocar incision or with morcellation. Pfannenstiel incision, often propagated for transperitoneal laparoscopic nephrectomy, can be an alternative for sample extraction opening the peritoneum close to the upper abdominal wall at the end of the procedure. Morcellation avoids incision augmentation but promotes longer operative time and less accuracy for the histopathology (20). We usually extract all samples by the initial trocar incision if possible or by making a small $5 \mathrm{~cm}$ inguinal incision (Gibson's incision) with low morbidity and good cosmetic results.

The retroperitoneal approach offers a lower morbidity when compared to the transperitoneal approach because it does not violate the peritoneal cavity. This benefit occurs not only due to less intraperitoneal manipulation and less organ lesions but also due to the reduction of the peritoneal contact with urine and blood $(5,7,13)$. Infected urine elimination during the surgery was observed in many patients in this series without any further complication such as infection or postoperative ileums.

Urology departments worldwide start their laparoscopic nephrectomy experience through transperitoneal access, which is considered the safest one and also the one that facilitates laparoscopic dissection especially in early experiences. This series shows the initial experience using retroperitoneal approach in a public Brazilian medical center without any previous familiarity with urological laparoscopic surgery. This experience demonstrates that the retroperitoneal approach is a safe, reproductive and effective access for simple nephrectomy.

\section{CONCLUSION}

The laparoscopic retroperitoneal approach for simple nephrectomy is a challenge for the surgeon due to a smaller surgical working space but on the other hand it is benefic to patients because it does not violate the peritoneal cavity. Retroperitoneal and transperitoneal laparoscopic approaches have the same surgical indications but previous lumbotomy is a relative contraindication for retroperitoneal access. Minimally invasive lumbar procedures such as nephrostomy and percutaneous nephrolithotripsy are not contraindications.

An advantage to retroperitoneoscopy is the straight access to the kidney and its renal pedicle making possible an easier and faster approach as well as vascular control. Disadvantages include the need to create a retroperitoneal space and a small working area to dissect, proceed reconstructive maneuvers and bag the sample to extract it out of the body.

Retroperitoneoscopy must be considered a safe, effective and reproductive surgical technique for nephrectomy in benign renal diseases.

\section{CONFLICT OF INTEREST}

None declared.

\section{REFERENCES}

1. Clayman RV, Kavoussi LR, Soper NJ, Dierks SM, Meretyk S, Darcy MD, et al.: Laparoscopic nephrectomy: initial case report. J Urol. 1991; 146: 278-82. 
2. Gaur DD, Agarwal DK, Purohit KC: Retroperitoneal laparoscopic nephrectomy: initial case report. J Urol. 1993; 149: 103-5.

3. Gaur DD: Laparoscopic operative retroperitoneoscopy: use of a new device. J Urol. 1992; 148: 1137-9.

4. Abbou CC, Cicco A, Gasman D, Hoznek A, Antiphon P, Chopin DK, et al.: Retroperitoneal laparoscopic versus open radical nephrectomy. J Urol. 1999; 161: 1776-80.

5. Desai MM, Strzempkowski B, Matin SF, Steinberg AP, Ng C, Meraney AM, et al.: Prospective randomized comparison of transperitoneal versus retroperitoneal laparoscopic radical nephrectomy. J Urol. 2005; 173: $38-41$.

6. Gupta NP, Goel R, Hemal AK, Dogra PN, Seth A, Aron $\mathrm{M}$, et al.: Should retroperitoneoscopic nephrectomy be the standard of care for benign nonfunctioning kidneys? An outcome analysis based on experience with 449 cases in a 5-year period. J Urol. 2004; 172: 14113.

7. Hoznek A, Salomon L, Gettman M, Stolzenburg JU, Abbou CC: Justification of extraperitoneal laparoscopic access for surgery of the upper urinary tract. Curr Urol Rep. 2004; 5: 93-9.

8. Rassweiler JJ, Seemann O, Frede T, Henkel TO, Alken P: Retroperitoneoscopy: experience with 200 cases. J Urol. 1998; 160: 1265-9.

9. Sebe P, de la Taille A, Hoznek A, Chopin D, Abbou CC, Salomon L: Simple nephrectomy with retroperitoneal laparoscopy. Prog Urol. 2003; 13: 577-80.

10. Hemal AK, Gupta NP, Kumar R: Comparison of retroperitoneoscopic nephrectomy with open surgery for tuberculous nonfunctioning kidneys. J Urol. 2000; 164: $32-5$

11. Zhang X, Zheng T, Ma X, Li HZ, Li LC, Wang SG, et al.: Comparison of retroperitoneoscopic nephrectomy versus open approaches to nonfunctioning tuberculous kidneys: a report of 44 cases. J Urol. 2005; 173: 1586-9.

12. Merrot T, Ordorica-Flores R, Steyeart H, Ginier C, Valla JS: Is diffuse xanthogranulomatous pyelonephritis a contraindication to retroperitoneoscopic nephroureterectomy? A case report. Surg Laparosc Endosc. 1998; 8: 366-9.

13. McDougall EM, Clayman RV: Laparoscopic nephrectomy for benign disease: comparison of the transperitoneal and retroperitoneal approaches. J Endourol. 1996; 10: 45-9.

14. Gill IS, Kavoussi LR, Clayman RV, Ehrlich R, Evans R, Fuchs G, et al.: Complications of laparoscopic nephrectomy in 185 patients: a multi-institutional review. J Urol. 1995; 154: 479-83.

15. Vallancien G, Cathelineau X, Baumert H, Doublet JD, Guillonneau B: Complications of transperitoneal laparoscopic surgery in urology: review of 1,311 procedures at a single center. J Urol. 2002; 168: 23-6.

16. Meraney AM, Samee AA, Gill IS: Vascular and bowel complications during retroperitoneal laparoscopic surgery. J Urol. 2002; 168: 1941-4.

17. Gill IS, Clayman RV, Albala DM, Aso Y, Chiu AW, Das S, et al.: Retroperitoneal and pelvic extraperitoneal laparoscopy: an international perspective. Urology. 1998; 52: 566-71.

18. Streich B, Decailliot F, Perney C, Duvaldestin P: Increased carbon dioxide absorption during retroperitoneal laparoscopy. Br J Anaesth. 2003; 91: 793-6.

19. Ng CS, Gill IS, Sung GT, Whalley DG, Graham R, Schweizer D: Retroperitoneoscopic surgery is not associated with increased carbon dioxide absorption. J Urol. 1999; 162: 1268-72.

20. Savage SJ, Gill IS: Intact specimen extraction during renal laparoscopy: muscle-splitting versus muscle-cutting incision. J Endourol. 2001; 15: 165-9.

\footnotetext{
Correspondence address:

Dr. Rodrigo Silva Quintela

Rua Correias 281 / 201

Belo Horizonte, MG, 30315-340, Brazil

E-mail: quintelarod@yahoo.com
} 


\section{EDITORIAL COMMENT}

It is a paper describing a retroperitoneal approach for nephrectomy in benign disease. Endoscopic nephrectomy is usually performed by transperitoneal rout and the merit of the paper is that it underlies the advantages of using a retroperitoneal approach and shows that it is feasible. One important shortcoming is the absence of a control group.

I believe that vessels should be ligated with a transfixational technique since it reduces the risk of intra and postoperative bleeding.
Conversion rate was fairly high. Manual dissection of the peritoneum gives a large working space. A hand-assisted technique also makes the operation easier, quicker and safer.

It could be argued that the total length of all incisions is longer than a small anterior extraperitoneal incision or dorsal incision for open surgery. The advantage with an endoscopic approach would be an extraction sit with low morbidity and good cosmetic results. A Pfannenstiel incision is therefore often propagated for the extraction (can also be used for a hand assisted procedure).

\author{
Dr. Jonas Wadström \\ Department of Surgical Sciences \\ Uppsala University Hospital \\ Uppsala, Sweden \\ E-mail: jonaswadstrom@akademiska.se
}

\section{EDITORIAL COMMENT}

Nowadays many authors in many countries including Japan have already described retroperitoneal nephrectomy for renal disease as a standard care. From this standpoint of view, the impact and origi-

nality of this manuscript is low internationally. However, as this may be a progressive approach in developing countries, this manuscript will be informative for many urologists working in these countries.

Dr. K. Mita

Hiroshima University, Dept of Urology Graduate School of Medical Sciences Hiroshima City, Japan E-mail:mita@plum.ocn.ne.jp

\section{EDITORIAL COMMENT}

The authors are to be congratulated on their excellent clinical results with retroperitoneoscopic nephrectomy.
In cases with giant hydronephrosis, I usually insert percutaneous nephrostomy intraoperativelly and adjust the volume of fluid within the collecting 
system to facilitate perirenal dissection in the retroperitoneal space.

In cases with horseshoe kidney, additional renal arteries may arise from the aorta or the iliac vessels. Using the retroperitoneal approach, it might be difficult to control hemorrhage or dividing the renal isthmus, especially when it is thick. Therefore, I prefer to use the transperitoneal approach in such cases.
Aside from early and straightforward access to the renal vessels, the retroperitoneal approach is not an easy operation because of a narrow working space and the relative paucity of anatomical landmarks. Although the authors successfully performed retroperitoneal nephrectomy despite of no previous experience with urologic laparoscopy, I think it is exceptional and inapplicable to general urologists. Educational training of laparoscopic surgery is desirable.

\section{Dr. A. Terai \\ Department of Urology \\ Kurashiki Central Hospital \\ Kurashiki, Japan \\ E-mail:at7899@kchnet.org.jp}

\section{EDITORIAL COMMENT}

This is a retrospective review of a clinical experience with retroperitoneal laparoscopy for benign kidneys at a public hospital. The authors adequately describe their technique. The results are in general favorable, but there were several open con- versions. Mostly for failure to progress from adhesions but there was at least one case of bleeding required open conversion. Complications were reported and discussed. I agree with the author's contention that the retroperitoneal technique is underutilized.

Dr. David A. Goldfarb

Division of Renal Transplant

Cleveland Clinic Foundation

Cleveland, Ohio, USA

E-mail: goldfad@ccf.org 